\title{
Mate recognition in fungi
}

\author{
LA Casselton \\ Department of Plant Sciences, University of Oxford, Oxford OX1 3RB, UK
}

\begin{abstract}
The ascomycete and basidiomycete fungi have contributed much to our understanding of eukaryotic cell biology. The study of mate recognition, in particular, has provided detailed understanding of cell signalling pathways and cellspecific gene transcription. Sexual dimorphism has little relevance to mating in these organisms, indeed specialised cells for mating are found only in filamentous ascomycetes and even here, a single individual produces both male and female structures. None the less, most species have genetic barriers to prevent selfing. The genes that determine self-
\end{abstract}

incompatibility divide populations into different mating types, and only individuals with different mating types can engage in sexual reproduction. Ascomycetes have just two mating types, but basidiomycetes may have several thousands. Despite apparent differences in the biology and numbers of mating types in these fungi, it is becoming increasingly apparent that many components of their mating pathways are highly conserved.

Heredity (2002) 88, 142-147. DOI: 10.1038/sj/hdy/6800035

Keywords: peptide pheromones; 7-TM receptors; MAP kinase pathways; HMG-domain; homeo-domain transcription factors

\section{Introduction}

The genes that determine fungal mating type are located at one or more complex loci, known as the mating type loci. Typical of these loci is that the DNA sequence is highly polymorphic. The term idiomorph is used to describe the situation in ascomycete fungi where alternative versions of the mating type locus on homologous chromosomes are completely dissimilar and encode unrelated proteins (Metzenberg and Glass, 1990). In the basidiomycetes, different mating types are conferred by different alleles of genes. Here highly polymorphic sequences are necessary to keep together a set of genes that encode essential proteins for mating but have evolved to be incompatible, so that compatible versions are only brought together by different mating types and not generated by intra-locus recombination (see Casselton and Olesnicky, 1998).

\section{Mating in yeasts}

The yeast life cycle is very simple because these ascomycete fungi are unicellular. Haploid cells have one of two mating types, designated MATa or MAT $\alpha$ in Saccharomyces cerevisiae, and (Plus) $P$ or (Minus) $M$ in Schizosaccharomyces pombe. Each haploid cell secretes a small peptide pheromone that binds compatible receptors on the surface of cells of the opposite mating type. Pheromone binding causes a characteristic response whereby cells reorientate growth towards the potential mating partner and then fuse to form a diploid cell. The diploid cell is

Correspondence: LA Casselton, Department of Plant Sciences, University of Oxford, South Parks Road, Oxford OX1 3RB, UK

E-mail: lorna.casselton@plants.ox.ac.uk no longer capable of mating but given the right environmental conditions, undergoes meiosis and sporulation.

The mating pathway of $S$. cerevisiae is the best understood of all fungi (see reviews by Herskowitz, 1988, 1989; Johnson, 1995). Unique MATa and MAT $\alpha$ DNA sequences encode transcription factors that permit the pheromone and receptor genes, amongst others, to be transcribed in a mating type-specific fashion. Two genes reside in each version of $M A T$ (Figure 1) but only three of these have known functions in mating, a1 at MATa and $\alpha 1$ and $\alpha 2$ at MAT $\alpha$. The a1 protein, a member of the homeodomain family of transcription factors, has no function in haploid cells; transcription of a cell-specific genes is activated by a general transcription factor belonging to the MADS box family (Mcm1p). In MAT $\alpha$ cells, $\alpha$ cell-specific genes must be activated and a cellspecific genes repressed. Transcription of $\alpha$ cell-specific genes occurs when Mcm1p binds co-operatively with the protein encoded by the $\alpha 1$ gene, and the protein encoded by the $\alpha 2$ gene, another homeodomain protein, is a repressor that prevents $\mathrm{Mcm} 1 \mathrm{p}$ activating transcription of a cell-specific genes.

Once compatible cells have fused, $\alpha 2$ continues to repress transcription of a cell-specific genes, and a new repressor complex, generated by heterodimerisation between the a1 and $\alpha 2$ proteins, prevents transcription of all genes required for general mating functions. This a1/ $\alpha 2$ heterodimer also represses transcription of $\alpha 1$, hence no $\alpha$ haploid cell-specific genes are expressed. Diploid cells become competent to undergo meiosis because the $a 1 / \alpha 2$ heterodimer is a repressor of a gene that inhibits initiation of meiosis (RME1).

Pheromone signalling enables cells of opposite mating type to detect each other (Leberer et al, 1997). Interestingly, in all ascomycetes, the pheromones synthesised by the alternative mating types belong to two distinct classes that are synthesised and secreted by different pathways 
Saccharomyces cerevisiae

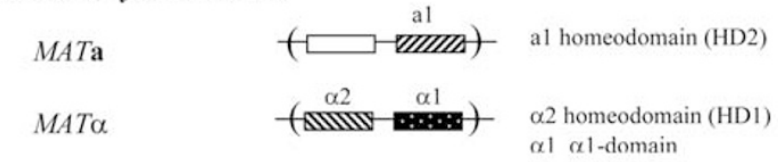

Schizosaccharomyces pombe

\begin{tabular}{|c|c|c|}
\hline mat $1-M$ & 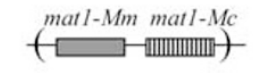 & $\begin{array}{l}\text { Mm unknown } \\
\text { Mc HMG domain }\end{array}$ \\
\hline mat $1-P$ & 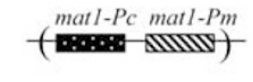 & $\begin{array}{l}\text { Pm homeodomain (HDl) } \\
\text { Pc } \alpha \text {-domain }\end{array}$ \\
\hline \multicolumn{3}{|c|}{ Ustilago maydis } \\
\hline mat $b$ & 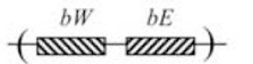 & $\begin{array}{l}\mathrm{bW} \text { homeodomain (HD2) } \\
\mathrm{bE} \text { homeodomain (HD1) }\end{array}$ \\
\hline mat a & pra & $\begin{array}{l}\text { Mfa pheromone } \\
\text { Pra receptor }\end{array}$ \\
\hline
\end{tabular}
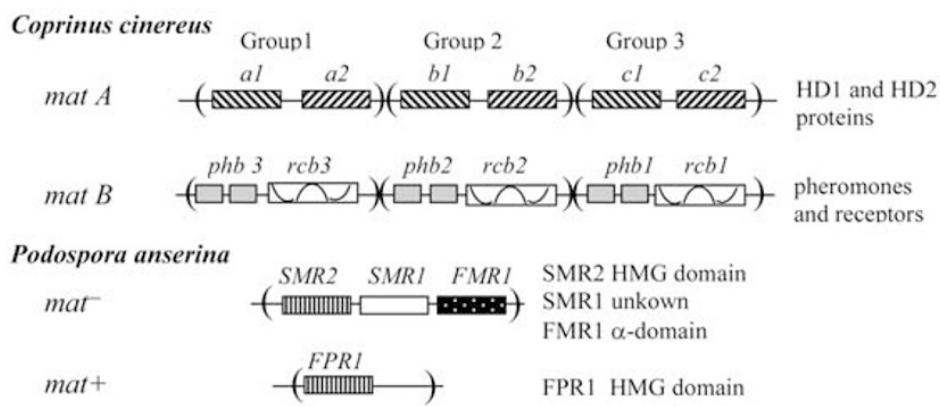
FACTOR
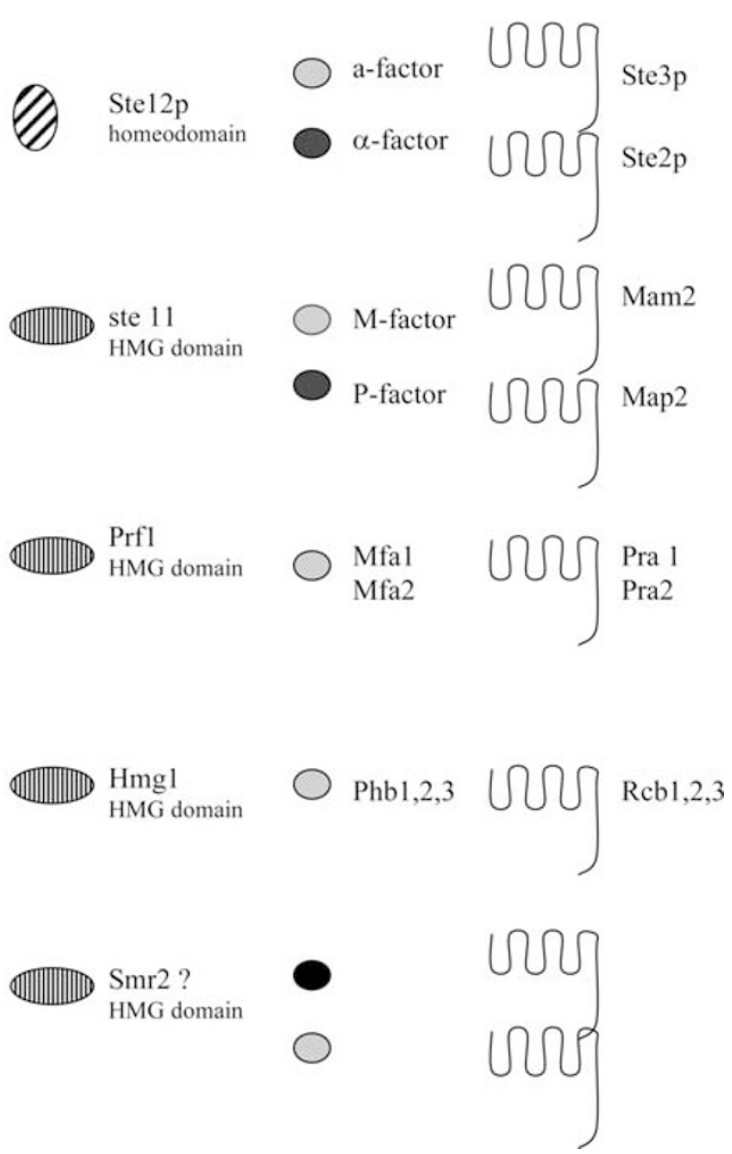

Figure 1 Essential proteins implicated in mating in ascomycete and basidiomycete fungi. Different motifs have been used to indicate that genes at the mating type loci encode proteins that have recognisable DNA-binding motifs such as the homeodomain, HMG domain and $\alpha$-domain. These same motifs are used to distinguish the two classes of proteins that co-ordinate the pheromone response. Different families of mating pheromones are distinguished by black and grey shading. Pheromone and receptor genes are predicted for $P$. anserina and have yet to be described.

(Caldwell et al, 1995). S. cerevisiae MATa cells make afactor, a 12 amino acid peptide that is processed from a much longer precursor molecule that has a CaaX motif at the C-terminus, a signal for carboxymethylation and farnesylation (Chen et al, 1997). The mature peptide is secreted via an ATP-binding cassette transporter (Ste6p). MAT $\alpha$ cells make $\alpha$-factor, which is a 13 amino acid peptide derived from precursors that have tandemly repeated copies of the sequence and this pheromone is secreted by the classical yeast secretory pathway. The significance of having these two classes of pheromone is not understood.

The pheromone receptors belong to the G-proteincoupled seven transmembrane-spanning domain family. Pheromone stimulation activates a mitogen activated protein (MAP) kinase cascade and the MAP kinase in this pathway (Fus3p), activates a target transcription factor known as Ste12p, another homeodomain protein, which regulates transcription of genes whose activity is co-ordinated for mating. The characteristic motif bound by Ste12p, the pheromone response element, is found in the promoters of these genes. Ste12p also binds the Mcm1p complex on a and $\alpha$ gene-specific promoters so that transcription is enhanced in response to pheromone stimulation and the pheromone signal is augmented.
Although mating in $S$. pombe is very similar to that in $S$. cerevisiae, there are interesting differences in the way the pathway is regulated, and the genes found at the mating type locus itself (Kelly et al, 1988; Nielsen and Davey, 1995). The Mat1-P locus resembles the MAT $\alpha$ locus of $S$. cerevisiae and has two genes that are homologues of $\alpha 1$ and $\alpha 2$ (mat1-Pc and mat1-Pm, respectively, Figure 1). There is no homologue of a1 at the alternative Mat1-M locus, instead, there is mat1-Mc, which encodes an HMG domain transcription factor and mat1-Mm, which encodes an as yet unknown class of protein.

Mating in S. pombe is induced by nutritional starvation. This leads to induction of the key transcriptional regulator, Ste11 (Sugimoto et al, 1991). Despite a similar function, Ste11 is not a homologue of S. cerevisiae Ste12p, it is another member of the HMG class of transcription factors. Ste11, once induced, autoregulates its own transcription (Kunitomo et al, 2000) and also induces enhanced levels of transcription of the mat1-Mc and mat1-Pc mating type genes and other genes required for mating.

As in S. cerevisiae, the genes present at the mating type locus ensure that the mating pheromones and receptors are expressed in a cell-specific manner. In $P$ cells, the $\alpha 1$ domain protein encoded by mat1-Pc acts co-operatively with an S. pombe Mcm1p homologue, Map1, to activate 
transcription of the $P$ cell pheromone and pheromone receptor genes (Nielsen et al, 1996), whereas in $M$ cells, the HMG domain protein encoded by mat1-Mc acts cooperatively with Ste11 to activate transcription of the corresponding $M$ cell genes (Kjaerulff et al, 1997). The other genes at the mating type locus, mat1-Pm and mat1-Mm are required to induce meiosis, and these are also induced by the pheromone signal. The pheromone signal thus promotes transcription of all four mating type genes and thereby co-ordinates the activities of genes necessary to initiate sexual development as well as those that bring mating partners together. The function of all four genes results in the expression of the mei3 gene, which encodes an inducer of meiosis (Willer et al, 1995).

\section{Mating in basidiomycetes}

The basidiomycete fungi are remarkable for having multiple mating types and for having a life cycle in which mating cell fusion and nuclear fusion are separated by an indefinite period of vegetative growth. The predominant vegetative phase in nature is the dikaryon, a filamentous mycelium in which the nuclei from compatible mating partners remain closely associated in each cell, and divide in synchrony but do not fuse (Figure 2). The dikaryon is, in effect, a continuous mating phase with the mating type genes playing a critical role in co-ordinating nuclear division and partitioning the two nuclei at each cell division. In many species a structure known as the clamp connection is involved in this partitioning (Figure 2). Nuclear fusion eventually occurs in specialised cells in differentiated fruiting structures, the mushroom for example, and is followed immediately by meiosis and sporulation. Familiar proteins involved in forming and maintaining the dikaryophase are homologues of the $S$. cerevisiae a1 and $\alpha 2$ homeodomain transcription factors and the various elements of the pheromone signalling pathway. Three species have been used as models to study mating in this group of fungi, the corn smut Ustilago maydis and the mushrooms Coprinus cinereus and Schizophyllum commune.

U. maydis has a unicellular asexual phase and, like yeasts, cells secrete mating pheromones that attract a compatible mating partner (see Banuett, 1995). Pheromone binding induces the formation of mating filaments that fuse at their tips to generate a cell that initiates growth of a filamentous dikaryon (Spellig et al, 1994). In mushroom species, the asexual stage is filamentous and is known as a monokaryon (homokaryon). Pheromones appear to have no role in cell fusion in mushroom species; this is mating type-independent. Pheromone signalling is activated after cell fusion and is necessary to initiate and maintain the dikaryophase (Olesnicky et al, 1999).

The mating type loci of $U$. maydis and $C$. cinereus are compared in Figure 1. In both species, there are two unlinked loci and compatible mates are those that have different alleles of genes at both (reviewed by Casselton and Olesnicky, 1998). One locus ( $b$ in $U$. maydis and $A$ in $C$. cinereus) contains genes encoding the homeodomain transcription factors (Gillissen et al, 1992; Kües et al, 1992). These genes are generally distinguished as HD1 ( $\alpha 2$ homologue) and HD2 (a1 homologue) based on the

a Podospora anserina: formation of the ascogenous hyphae

fertilisation
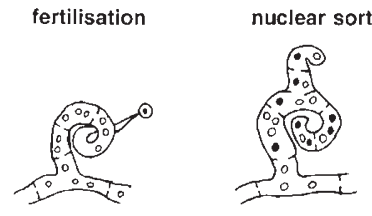

b Coprinus cinereus: formation of the dikaryon

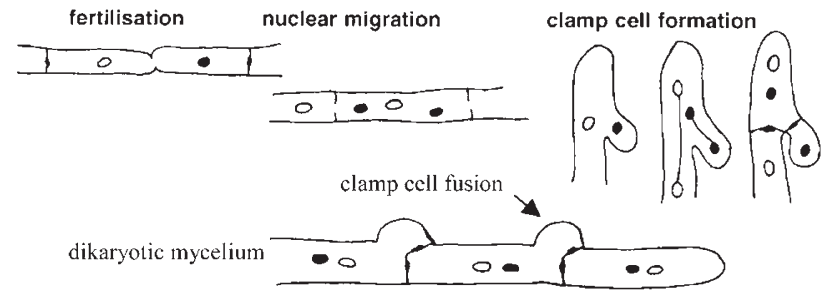

Figure 2 Parallel events in the initiation of a dikaryophase in (a), the filamentous ascomycete, Podospora anserina, and (b), the basidiomycete, Coprinus cinereus. In P. anserina, fusion occurs between a male microconidium and the tip of the female ascogonial hypha. In C. cinereus, fusion is between vegetative hyphal cells. The donor nucleus migrates within the accepting hypha and eventually binucleate cells emerge containing a nucleus from each mate. The hyphal tip cell forms a hook, termed the crozier in P. anserina and the clamp cell in C. cinereus, and one of the nuclei divides within this cell. The crozier and the clamp cell fuse (arrowed) with the newly formed subterminal cell to restore a binucleate cell organisation. The new tip cell in the ascogenous hypha becomes an ascus in which nuclear fusion, meiosis and sporulation occurs and the dikaryophase is proliferated from the subterminal cell. In $C$. cinereus, the tip cell continues to divide indefinitely to give a vegetative dikaryotic mycelium with meiosis and sporulation delayed until the formation of the mushroom fruiting body.

conserved but clearly different homeodomain sequences (Kües and Casselton, 1992). As in S. cerevisiae, the two classes of proteins heterodimerise in a compatible mating to generate a transcription factor that in basidiomycetes is unique to dikaryotic cells. At the second mating type locus ( $a$ in $U$. maydis, $B$ in C. cinereus) are genes encoding the mating pheromones and receptors, which, in this group of fungi have been recruited to act directly as mating type determinants (Bölker et al, 1992; Wendland et al, 1995).

$U$. maydis has some 50 different mating types (see, Banuett, 1995). The $a$ locus has just two allelic versions, a1 and $a 2$ each containing a receptor and a pheromone gene (Bölker et al, 1992) (Figure 1), but there are at least 25 allelic versions of the $b$ locus, which contains a single HD1 and HD2 gene (Gillissen et al, 1992) (Figure 1). The thousands of mating types we see in mushrooms have been generated by gene duplication. In $C$. cinereus, for example, instead of one pair of homeobox genes, there are now three (Pardo et al, 1996), and instead of one receptor and associated pheromone gene(s), there are three (O'Shea et al, 1998; Halsall et al, 2000). There are three paralogous groups of genes that encode functionally redundant proteins (Figure 1). All the genes are multiallelic and over the course of evolution, there has been recombination between the different groups to 
generate all possible combinations of their different allelic versions (May and Matzke, 1995). Just five allelic versions of each group would be sufficient to generate $(5 \times 5 \times 5)$ 125 unique $A$ and $B$ mating specificities. In $C$. cinereus there are an estimated $160 A$ and $79 B$ specificities, which together generate $>12000$ mating types (Raper, 1966). Not all mating types are compatible, because some will be identical for $A$ and some for $B$, but more than $90 \%$ of chance encounters will generate dikaryons. Even larger numbers of $A$ and $B$ gene alleles in $S$. commune generate more than 20000 mating types (Raper, 1966). In this species, recombination occurs regularly between the paralogous groups of genes because these are separated by homologous DNA sequence.

The evolution of multiple mating types has imposed strong selection for highly specific protein-protein interactions that are not necessary where only two mating types exist. Both classes of homeodomain proteins are present in unmated cells encoded by genes at the same mating type locus, and these are unable to heterodimerise to form an active transcription factor. Dimerisation is only possible when proteins encoded by different allelic versions of the genes are brought together on mating. A discriminating dimerisation domain within their $\mathrm{N}$ terminal domains permits only compatible HD1 and HD2 proteins to form an active transcription factor complex (Banham et al, 1995; Kämper et al, 1995; Magae et al, 1995) that can localise to the nucleus (Spit et al, 1998). The mushroom species appear to be unique in having large families of receptors and pheromones. These molecules also display remarkable specificity. None of the receptors are activated by pheromones encoded by genes within the same locus, only different allelic versions of pheromones and receptors brought together on mating are able to activate each other (Olesnicky et al, 2000).

As in the yeasts, pheromone binding to compatible receptors activates a MAP kinase cascade, several components of which have been identified in $U$. maydis. Significantly the target transcription factor of this pathway, termed Prf1, appears to be a homologue of the $S$. pombe HMG protein Ste11 (Hartmann et al, 1996, 1999). Like the ste11 gene, prf1 is induced by nutritional starvation, and once induced, Prf1 autoregulates its own transcription in response to pheromone stimulation. As in S. pombe, the pheromone signal co-ordinates the activities of all the mating type genes in $U$. maydis (Hartmann et al, 1996; Urban et al, 1996); it leads to enhanced transcription of the pheromone and receptor genes at the $a$ locus and induces transcription of the homeobox genes at the $b$ locus.

Interestingly, only pheromones belonging to the $S$. cerevisiae a-factor class are found in basidiomycetes (Bölker and Kahmann, 1993; Vaillancourt and Raper, 1996). Despite having no obvious sequence similarity to a-factor precursor, pheromone genes from both $C$. cinereus and $S$. commune can be expressed in $S$. cerevisiae cells and secreted as peptides capable of activating a compatible mushroom receptor also expressed in yeast cells (Fowler et al, 1999; Olesnicky et al, 1999). Pheromone processing, as expected, appears to be MATa cell-dependent, indicating that processing is via the normal a-factor maturation pathway and that this pathway is highly conserved in what are quite distantly related fungi (Olesnicky et al, 1999).

\section{Mating in filamentous ascomycetes}

In species such as Neurospora crassa and Podospora anserina, two model species, mating is initiated between differentiated male and female cells, the microconidium and the ascogonium, respectively. The microconidium fuses with the trichogyne, the receptive tip of the ascogonial hypha, and its nucleus replicates with resident nuclei (Figure 2). Nuclear sorting follows to generate typically binucleate dikaryotic cells, the ascogenous hyphae, in which synchronised nuclear division occurs and daughter nuclei are partitioned via a structure analogous to the clamp connection seen in basidiomycete dikaryons. Thus filamentous ascomycetes, like the basidiomycetes, delay nuclear fusion and proliferate the nuclei of a mating pair with a dikaryophase. This phase is of limited duration in ascomycetes and is confined within the developing fruiting body. Pheromone signalling has long been implicated in the attraction of microconidia to the trychogyne (Bistis, 1983), but it is only recently that the genes encoding pheromones have been identified in this group of fungi (Zhang et al, 1998; Shen et al, 1999; Pöggeler, 2000). The similarities between the ascogenous hyphae and the basidiomycete dikaryon illustrated in Figure 2 clearly point to strong conservation in the way the mating pathways of these fungi are regulated, but this is not immediately obvious when one looks at the genes sequestered at the mating type locus!

The idiomorphs of the P. anserina and N. crassa mating type locus contain similar genes (Debuchy et al, 1993; Ferreira et al, 1996; reviewed by Coppin et al, 1997); those of $P$. anserina are designated $\mathrm{mat}^{-}$and $\mathrm{mat}^{+}$loci and are illustrated in Figure 1. Two genes have been shown to be essential for fertilisation, FMR1 in the mat ${ }^{-}$idiomorph and FPR1 in the $\mathrm{mat}^{+}$idiomorph. Interestingly these two genes are the only genes to be found in the idiomorphs of less closely related species (Turgeon et al, 1993; Turgeon, 1998). Both genes have obvious homologues in the yeast mating type loci. FMR1 encodes a homologue of $S$. cerevisiae $\alpha 1$ and S. pombe Mat1-Mc, a protein that is presumably required to activate the transcription of $\mathrm{mat}^{-}$ cell-specific mating pheromones and receptors. FPR1 encodes a protein belonging to the HMG domain family and is the likely homologue of the Mat1-Pc protein of $S$. pombe, and required to regulate transcription of $\mathrm{mat}^{+}$cellspecific mating pheromone and receptor genes. The two additional genes in the mat $^{-}$idiomorph encode proteins that are essential for completing sexual development, but these are only induced during mating (Coppin and Debuchy, 2000). SMR2 encodes another member of the HMG domain family and is required to maintain proper nuclear pairing in the ascogenous hyphae (Zickler et al, 1995), which by analogy to the dikaryon of basidiomycetes is predicted to be dependent on the pheromone signal. It seems likely that $S M R 2$ is the P. anserina homologue of $S$. pombe ste11 and $U$. maydis prf1. There is no obvious advantage in having this gene sequestered into a mating type locus, as evidenced by the fact that not all filametous ascomycetes do so, but the fact that it can be indicates that its function is required only after cell fusion.

\section{Concluding comments}

Sexual development requires the co-ordinated activities of a series of transcriptional regulators. By separating the 
genes that encode one or more of these into idiomorphic mating type loci, mating becomes essential in order to complete the necessary regulatory network. Amongst ascomycetes, different genes have been elected to fulfil the mating type role. Where there are multiple mating types in basidiomycetes, there is no longer the need for the mating type genes to differentially regulate gene transcription, hence no role for proteins like $\alpha 1$. A different strategy has evolved to prevent selfing. Only proteins that interact with another protein are encoded at the mating type loci and these have evolved to be so specific in their interactions that only proteins from different mates can activate sexual development. HMG proteins play a prominant role in mate recognition and sexual development in all but $S$. cerevisiae. Recent work on $C$. cinereus has implicated two HMG proteins in mating, Hmg1 (author's unpublished data) and Pcc1 (Murata et al., 1998), both of which, like their counterparts in S. pombe and P. anserina, are required for successful pheromone signalling. Significant or not, this family of proteins also plays a key role in sex determination in mammals! (Sinclair et al, 1990). Studies on the fungal homeodomain mating proteins have contributed immensely to our understanding of the ways in which these transcription factors acquire their specificity (Johnson, 1995; Li et al, 1995). Similar studies on the fungal HMG mating proteins promise to be equally rewarding (van Beest et al, 2000).

\section{Acknowledgements}

Work on Coprinus cinereus has been supported by BBSRC and the Gatsby Charitable Foundation.

\section{References}

Banham AH, Asante-Owusu RN, Göttgens B, Kingsnorth CS, Thompson SAJ, Mellor EJC et al (1995). An N-terminal dimerization domain permits homeodomain proteins to choose compatible partners and initiate sexual development in the mushroom Coprinus cinereus. Plant Cell 7: 773-783.

Bannuett F (1995). Genetics of Ustilago maydis, a fungal pathogen that induces tumours in maize. Annu Rev Genet 29: 179-208.

Bistis GN (1983) Evidence for diffusible, mating-type-specific trichogyne attractants in Neurospora crassa. Exp Mycol 7: 292-295.

Bölker M, Urban M, Kahmann R (1992). The $a$ mating type locus of $U$. maydis specifies cell signaling components. Cell 68: 441-450.

Bölker M, Kahmann R (1993). Sexual pheromones and mating responses in fungi. Plant Cell 5: 1461-1469.

Caldwell GA, Naider F, Becker JM (1995). Fungal lipopeptide mating pheromones: a model system for the study of protein prenylation. Microbiol Rev 59: 406-422.

Casselton LA, Olesnicky NL (1998). Molecular genetics of mating recognition in basidiomycete fungi. Microbiol Mol Biol Rev 62: $55-70$

Chen P, Sapperstein SK, Choi JC, Michaelis S (1997). Biogenesis of the Saccharomyces cerevisiae mating pheromone a-factor. J Cell Biol 136: 251-269.

Coppin E, Debuchy R (2000). Co-expression of the mating-type genes involved in nuclear recognition is lethal in Podospora anserina. Genetics 155: 657-669.

Coppin E, Debuchy R, Arnaise S, Picard M (1997). Mating types and sexual development in filamentous ascomycetes. Microbiol Mol Biol Rev 61: 411-428.

Debuchy R, Arnaise S, Lecellier G (1993) The mat- allele of Podospora anserina contains three regulatory genes required for the development of fertilized female organs. Mol Gen Genet 241: 667-673.

Ferreira AVB, Saupe S, Glass NL (1996) Transcriptional analysis of the $m t A$ idiomorph of Neurospora crassa identifies two genes in addition to $m t A-1$. Mol Gen Genet 250: 767-774.

Fowler TJS, DeSimone SM, Mitton MF, Kurjan J, Raper CA (1999). Multiple sex pheromones and receptors of a mushroom-producing fungus elicit mating in yeast. Mol Biol Cell 10: 2559-2572

Gillissen B, Bergemann J, Sandmann C, Schroeer B, Bölker M, Kahmann R (1992) A two-component regulatory system for self/nonself recognition in Ustilago maydis. Cell 68: 647-657.

Halsall JR, Milner MJ, Casselton LA (2000) Three subfamilies of pheromone and receptor genes generate multiple $B$ mating specificities in the mushroom Coprinus cinereus. Genetics 154: $115-1123$.

Hartmann HA, Kahmann R, Bölker M (1996). The pheromone response factor coordinates filamentous growth and pathogenicity in Ustilago maydis. EMBO J 15: 1632-1641.

Hartmann HA, Krüger J, Lottspeich F, Kahmann R (1999). Environmental signals controlling sexual development of the corn smut fungus Ustilago maydis through the transcriptional regulator Prf1. Plant Cell 11: 1293-1305.

Herskowitz I (1988). Life cycle of the budding yeast Saccharomyces cerevisiae. Microbiol Rev 52: 536-553.

Herskowitz I (1989). A regulatory hierarchy for cell specialization in yeast. Nature 342: 749-757.

Johnson AD (1995). Molecular mechanisms of cell-type determination in budding yeast. Curr Opin Genet Dev 5: 552-558.

Kämper J, Reichmann M, Romeis T, Bölker M, Kahmann R (1995). Multiallelic recognition, nonself-dependent dimerization of the $\mathrm{bE}$ and $\mathrm{bW}$ homeodomain proteins in Ustilago maydis. Cell 81: 73-83.

Kjaerulff S, Dooijes D, Clevers H, Nielsen O (1997). Cell differentiation by interaction of two HMG-box proteins: Mat1-Mc activates $M$-cell-specific genes in $S$. pombe by recruiting the ubiquitous transcription factor Ste11 to weak binding sites. EMBO J 13: 4021-4033.

Kelly M, Burke J, Smith M, Klar A, Beach D (1988). Four matingtype genes control sexual differentiation in the fission yeast. EMBO J 7: 1537-1547.

Kües U, Casselton LA (1992). Homeodomains and regulation of sexual development in basidiomycetes. Trends Genet 8: 154155.

Kües U, Richardson WVJ, Tymon AM, Mutasa ES, Göttgens B, Gaubatz $\mathrm{S}$ et al (1992). The combination of dissimilar alleles of the $A \alpha$ and $A \beta$ gene complexes, whose proteins contain homeodomain motifs, determine sexual development in the mushroom Coprinus cinereus. Genes Dev 4: 568-577.

Kunitomo H, Higuchi T, Iino Y, Yamamoto M (2000). A zinc finger protein, Rst2p, regulates transcription of the fission yeast ste11+ gene, which encodes a pivotal transcription factor for sexual development. Mol Biol Cell 11: 3205-3217.

Leberer E, Thomas DY, Whiteway M (1997). Pheromone signalling and polarized morphogenesis in yeast. Curr Opin Genet Dev 7: 59-66.

Magae Y, Novotny C, Ullrich R (1995). Interaction of the $A$ alpha $Y$ mating-type and $Z$ mating-type homeodomain proteins of Schizophyllum commune detected by the two-hybrid system. Biochiem Biophys Res Commun 211: 1071-1076.

May G, Matzke E (1995). Recombination and variation at the $A$ mating-type locus of Coprinus cinereus. Mol Biol Evol 12: 794-802.

Metzenberg RL, Glass NL (1990). Mating type and mating strategies in Neurospora. BioEssays 12: 53-59.

Murata Y, Fujii M, Zolan ME, Kamada T (1998). Molecular analysis of $p c c 1$, a gene that leads to $A$-regulated sexual morphogenesis in Coprinus cinereus. Genetics 149: 1753-1761.

Nielsen O, Davey J (1995). Pheromone communication in the fission yeast Schizosaccharomyces pombe. Semin Cell Biol 6: 95104 
Nielsen O, Friis T, Kjaerulff S (1996). The Schizosaccharomyces pombe map1 gene encodes an SRF/MCM1-related protein required for $P$ cell-specific gene expression. Mol Gen Genet 253: 387-393.

Olesnicky NS, Brown AJ, Dowell SJ, Casselton LA (1999). A constitutively active G-protein-coupled receptor causes mating self-compatibility in the mushroom Coprinus. EMBO J 18: 2756-2763.

Olesnicky NS, Brown AJ, Honda Y, Dyos SL, Dowell SJ, Casselton LA (2000). Self-compatible B mutants in Coprinus with altered pheromone-receptor specificities. Genetics 156: 10251033.

O'Shea SF, Chaure PT, Halsall JR, Olesnicky,NS, Leibbrant A, Connerton IF et al (1998). A large pheromone and receptor gene complex determines multiple $B$ mating specificities in Coprinus cinereus. Genetics 148: 1081-1090.

Pardo EH, O'Shea SF, Casselton LA (1996). Multiple versions of the $A$ mating type locus of Coprinus cinereus are generated by three paralogous pairs of multiallelic homeobox genes. Genetics 144: 87-94.

Pöggeler S (2000). Two pheromone precursor genes are transcriptionally expressed in the homothallic ascomycete Sordaria macrospora. Curr Genet 37: 403-411.

Raper JR (1966). Genetics of Sexuality in Higher Fungi. The Ronald Press Company: New York.

Shen WC, Bobrowicz P, Ebbole DJ (1999). Isolation of pheromone precursor genes of Magnoporthe grisea. Fungal Genet Biol 27: 253-263.

Sinclair AH, Berta P, Palmer MS, Hawkins JR, Griffiths BL, Matthijs JS et al (1990). A gene from the human sex-determining region encodes a protein with homology to a conserved DNAbinding motif. Nature 34: 240-244.

Spellig T, Bölker M, Lottspeich F, Frank RW, Kahmann R (1994). Pheromones trigger filamentous growth in Ustilago maydis. EMBO J 13: 1620-1627.

Spit A, Hyland R, Mellor EJC, Casselton LA (1998). Heterodimerization targets a homeodomain protein complex to the nucleus. Proc Natl Acad Sci USA 95: 6228-6233.

Sugimoto A, Iino Y, Maeda Y, Watanabe Y, Yamamoto M (1991). Schizosaccharomyces pombe ste11+ encodes a transcription factor with an HMG motif that is a critical regulator of sexual development. Gene Dev 5: 1990-1999.

Turgeon BG (1998). Application of mating type gene technology to problems in fungal biology. Annu Rev Phytopathol 36: 115-137.

Turgeon BG, Bohlmann H, Ciuffetti LM, Christiansen SK, Yang G, Schäfer W et al (1993). Cloning and analysis of the matingtype genes from Cochliobolus heterostrophus. Mol Gen Genet 238: 270-284.

Urban M, Kahmann R, Bölker M (1996). Identification of the pheromone response elements in Ustilago maydis. Mol Gen Genet 251: 31-37.

Vaillancourt LJ, Raper CA (1996). Pheromones and pheromone receptors as mating-type determinants in basidiomycetes. In: Setlow JK (ed) Genetic Engineering Vol. 18, Plenum Press: New York. pp 219-247.

van Beest M, Dooijes D, van de Wetering M, Kjaerulff S, Bonvin A, Nielsen $O$ et al (2000). Sequence-specific high mobility group box factors recognize 10-12-base pair minor goove motifs. J Biol Chem 275: 27266-27273.

Wendland JL, Vaillancourt LJ, Hegner J, Lengeler KB, Laddison KJ, Specht CA et al (1995). The mating-type locus B $\alpha 1$ of Schizophyllum commune contains a pheromone receptor gene and putative pheromone genes. EMBO J 14: 5271-5278.

Willer M, Hoffman 1, Styrarsdottir U, Egel R, Davey J, Nielsen O (1995). Two-step activation of meiosis by the mat1 locus in Schizosaccharomyces pombe. Mol Cell Biol 15: 4964-4970.

Zickler D, Arnaise S, Coppin E, Debuchy R, Picard M (1995). Altered mating-type identity in the fungus Podospora anserina leads to selfish nuclei, uniparental progeny, and haploid meiosis. Genetics 140: 493-503.

Zhang L, Baasiri RA, van Alfen KK (1998). Viral repression of the fungal pheromone-precursor gene expression. Mol Cell Biol 18: 953-959. 